\title{
Flying Capacitor DTC Drive with Reductions in Common Mode Voltage and Stator Overvoltage
}

\author{
Abdolreza Rahmati ${ }^{\dagger}$, Mohammad Arasteh*, Shahrokh Farhangi**, and Adib Abrishamifar* \\ $\dagger^{*}$ Dept. of Electrical Engineering, Iran University of Science and Technology, Tehran, Iran \\ ** School of Electrical and Computer Engineering, University of Tehran, Tehran, Iran
}

\begin{abstract}
This paper gives a detailed analysis of the direct torque control (DTC) strategy in a five-level drive and proposes a 24-sector switching table. The known problems in low-voltage drives such as bearings currents and an overvoltage phenomenon which leads to premature failure are reviewed and the occurrence of these problems in medium voltage drives has been investigated. Then a solutions to these problems is presented and the switching table to deal with these problems is modified. Simulation and experimental results on a $3 \mathrm{kVA}$ prototype confirm the proposed solution. In implementing the above strategy a TMS320F2812 is used.
\end{abstract}

Key Words: Common mode voltage, Direct torque control, Multilevel flying capacitor drive, Stator overvoltage

\section{INTRODUCTION}

Nowadays, high power medium voltage drives are widely used in petrochemical, transportation, cement and steel industries. Research in the field of improving performance and increasing the power of these medium voltage drives has increased and in the last decade it has become one of the most active areas of power electronics. Development of these drives began in the last decade using GTOs, while today IGCTs and high voltage IGBTs with their improved switching features, simplicity of control and low losses are prominent choices [1]. Although numerous structures have been developed for medium voltage drives, three structures including diode-clamped, H-bridge and flying capacitor find greater application and are commercially available [2]-[5]. Among the above structures, the flying capacitor inverter, as shown in Fig. 1, is noteworthy because, unlike the H-bridge inverter, it does not require a transformer, and because it does not have the problems, such as reverse recovery currents and capacitor voltage balancing, found in diode-clamped inverters [2]. It is even more attractive at high switching frequencies where it leads to low capacitor values. Among the methods of induction motor speed control, a DTC strategy with a high performance level in low-voltage drives, has also been implemented in multilevel drives [6]-[9].

The DTC strategy in [9] has been implemented, but a detailed analysis of the strategy was not provided. Also problems

\footnotetext{
Manuscript received Dec. 20, 2010; revised Mar. 13, 2011

Recommended for publication by Associate Editor Yong-Sug Suh.

$\dagger$ Corresponding Author: rahmati@iust.ac.ir

Tel: +9821-77-240-492, Fax: +9821-77-240-490, IUST

* Dept. of Electrical Engineering, Iran University of Science and Technology, Iran

${ }^{* *}$ School of Electrical and Computer Engineering, University of Tehran, Iran
}

such as common mode voltage (CMV) and the overvoltage which causes premature failure in electrical motors have not been addressed.

The invention of IGBTs with a rise time in the range of 50ns has played a major role in the development of PWM drives. However, this fast rise time may cause an overvoltage at the motor terminals, which can be several times the DC supply voltage. It can also cause non-uniform voltage distribution in the stator winding. Due to this overvoltage, stator insulation to the ground and insulation between the winding turns will be stressed. This leads to premature motor failure primarily because the motor's insulations are designed to work in the range of the rated voltage and frequency. Factors that affect the overvoltage include the cables, the motor and cable impedance matching, the inverter output $d v / d t$ and the modulation strategy [10], [11]. So far several solutions to overcome these problems in low voltage drives have been presented. These can be divided into five categories: increasing the rise time, using an inverter output filter or an impedance matching circuit in the motor, using appropriate cables, increasing the motor tolerance and modulation correction. One advantage of multilevel converters is that the input voltage is divided by the number of levels and at every moment a fraction of the DC voltage is switched. Thus the voltage reflection will correspond to the same changes. In other words, $d v$ is decreased and the motor overvoltage is divided by the number of levels in a multilevel inverter. With a reduced $d v$ and an increased $d t$, the overvoltage problem caused by the reflected voltage in multilevel drives with a long cable is less than that experienced with two-level drives.

Another issue in PWM-drives is a resonance phenomena that leads to motor overvoltage and thus to premature failure 


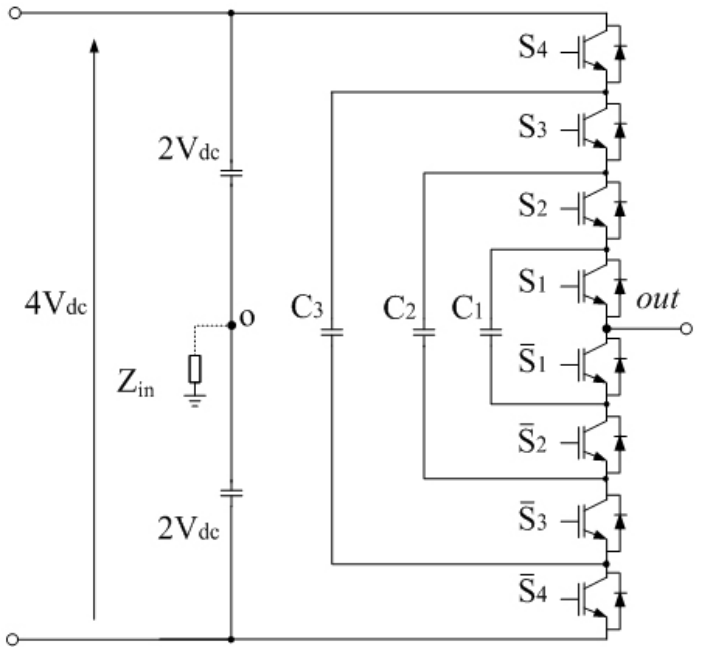

Fig. 1. One leg of a 3-phases flying capacitor inverter.

[12], [13]. Overvoltage due to this resonance phenomena requires different solutions. In [12] insulation failure, due to the resonant frequencies that exist between the motor and the transformer inductances with the cable capacitance, is prevented by installing a low pass filter (LCR filter). In [13] by increasing the switching frequency above the resonant frequency, the stator overvoltage is prevented.

The bearing current caused by the breakdown of the thin layers of grease (electrical discharge machining) is the main factor in bearing damage and is a function of the CMV [14]. Several methods for dealing with the bearing current in low voltage drives are provided and can be classified into two groups: bearing current elimination techniques and methods of bearing current reduction. These methods require a special motor or are expensive. All methods of reducing the CMV on low voltage motors are applicable to medium voltage motors.

\section{Direct Torque Control of Multilevel Drives}

This section is devoted to an analysis of DTC for a five level drive.

\section{A. Principles}

In a three-phase symmetrical induction motor, the electromagnetic torque can be expressed by:

$$
T_{e}=\frac{3}{2} p \frac{L_{m}}{\sigma L_{s} L_{r}}\left|\bar{\psi}_{s}\right|\left|\bar{\psi}_{r}\right| \operatorname{Sin} \delta_{\psi}
$$

where $\left|\bar{\psi}_{s}\right|$ and $\left|\bar{\psi}_{r}\right|$, are the stator and rotor flux amplitudes respectively and $\delta_{\psi}$, the angle between the two flux vectors, is the torque angle. $L_{m}, L_{s}$ and $L_{r}$ are the magnetizing, stator and rotor inductances, respectively and $\sigma$ is the total leakage factor of the motor defined as:

$$
\sigma=1-\frac{L_{m}^{2}}{L_{s} L_{r}}
$$

In DTC, the flux amplitude is maintained at about a nominal value and the torque angle is used to control the torque according to (1). Obviously, the maximum torque is achieved for $\delta \psi=\pi / 2$. On the other hand, the torque angle is adjustable by using the stator voltage vector. The stator voltage equation is given by:

$$
\bar{u}_{s}=r_{s} \bar{i}_{s}+\frac{d \bar{\psi}_{s}}{d t}
$$

(3) can be rewritten as:

$$
\frac{d \bar{\psi}_{s}}{d t}=\bar{u}_{s}-r_{s} \bar{i}_{s}
$$

In multilevel drives, like two-level drives, the stator flux trajectory can be controlled by selecting appropriate stator voltage vectors. Let the rotating reference system align with the stator flux vector as shown in Fig. 2, then $\left|\bar{\psi}_{s}\right|$ (hence $\left.\left|\bar{\psi}_{r}\right|\right)$ and $\angle \bar{\psi}_{s}$ can be controlled by the $d$ - and $q$-components of $\bar{u}_{s}$, respectively. In other words, the modulus of the stator flux is affected by $u_{s d}$ and angle of the stator flux vector is affected by $u_{s q}$. For instance, applying the $\bar{u}_{s 1}$ and $\bar{u}_{s 2}$ vectors shown in Fig. 2, letting $u_{s q 1}=u_{s q 2}$, and applying $\bar{u}_{s 1}$ and $\bar{u}_{s 2}$ generate the same torque. Although both vectors increase the flux amplitude, $\bar{u}_{s 2}$ leads to a faster variation in the modulus of the stator flux, since $u_{s d 2}>u_{s d 1}$. On the other hand, the torque angle depends on the angle of the rotor flux. The change of the rotor flux position angle related to the stator current and the rotor speed is given by [19]:

$$
\frac{d \angle \bar{\psi}_{r}}{d t}=\omega+\frac{L_{m}}{T_{r}\left|\bar{\psi}_{r}\right|} i_{s q}
$$

where $\angle \bar{\psi}_{r}$ is the position angle of $\bar{\psi}_{r}, \omega$ is the rotor electrical speed, $T_{r}$ is the rotor time constant, and $i_{s q}$ is the $q$-component of $\bar{i}_{s}$ with respect to the stator flux axis. $\bar{u}_{s}$ develops $i_{s q}$ with a delay related to the leakage time constant of the stator while the mechanical time constant of the drive inhibits a fast change of $\omega$. For instance, at medium speeds a medium-size $u_{s q}$ can increase the torque angle and consequently the amount of torque, while at high speeds the same $u_{s q}$ causes the torque to be reduced. Therefore vector selection is related to the rotor speed. Since there are four hexagons in the $\alpha \beta$-plane, from the zero speed to the nominal speed can be divided into four ranges as (6): very low, low, medium and high speeds $\left(\omega_{m}\right.$ is the rotor angular speed and $\omega_{\text {nom }}$ is nominal rotor angular speed):

$$
\left\{\begin{array}{l}
0 \leq \omega_{m}<\omega_{\text {nom }} / 4 \\
\omega_{\text {nom }} / 4 \leq \omega_{m}<\omega_{\text {nom }} / 2 \\
\omega_{\text {nom }} / 2 \leq \omega_{m}<3 \omega_{\text {nom }} / 4 \\
3 \omega_{\text {nom }} / 4 \leq \omega_{m} \leq \omega_{\text {nom }}
\end{array}\right.
$$

\section{B. Switching Table Design}

The stator voltage vector in a multilevel drive is given by the well-known relation:

$$
\bar{u}_{s}=u_{a} e^{j 0}+u_{b} e^{j 2 \pi / 3}+u_{c} e^{j 4 \pi / 3}
$$

This space voltage vector can be indicated as a number cba in which a, b and c, respectively, show the voltage levels in the related phases. All of the space voltage vectors for a fivelevel drive except for the redundant vectors are shown in Fig. 3. Compared to two-level inverters, multilevel inverters have a high number of voltage vectors with different sizes so that the flux vector can be accurately controlled. Therefore, the 


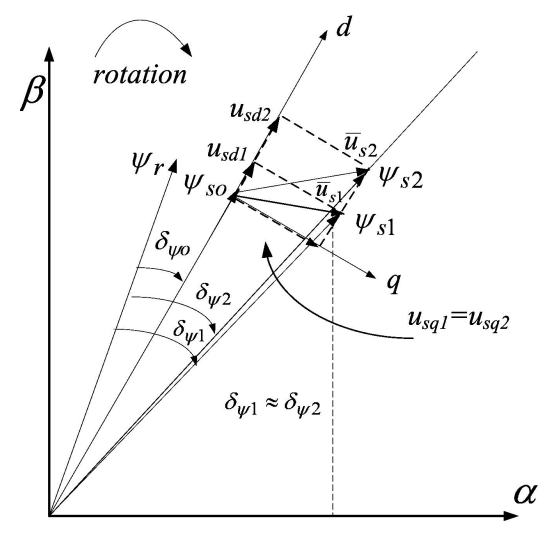

Fig. 2. Impact of stator voltage vectors on torque.

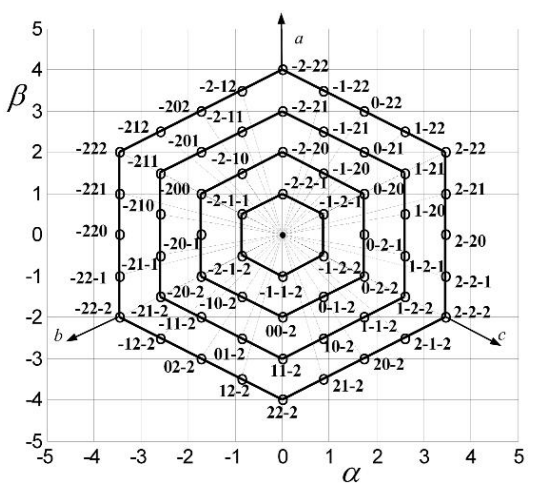

Fig. 3. Voltage space vectors in a five-level inverter.

multilevel drives have a faster and more precise control over the flux and torque.

As shown in Fig. 3, the voltage vectors are inscribed in four hexagons. Since in the outer hexagon there are 24 voltage vectors, in this paper the $\alpha \beta$-plane is divided into 24 sectors as shown in Fig.4. Flexibility and only a one-level transition during sector variation are the important advantages of a 24-sector switching table. In order to design this switching table, the impact of different voltage vectors on the torque is investigated. Here, the switching table design at high speeds is presented. Assume that the stator flux vector is located in sector $\theta 1$ along the $a$-axis. In this case, all of the vectors in $\alpha>0, \beta \geq 0$ increase the flux amplitude and all of the vectors in the region $\alpha>0, \beta<0$ cause the flux amplitude to decrease. Among these vectors, the group vectors $0-2 \mathrm{x}, 1-$ $2 \mathrm{x}$ and 2-2x, respectively, result in a small, medium and large variations in the stator flux angle $\angle \bar{\psi}_{s}$. Therefore, these groups can decrease, keep constant or increase the electromagnetic torque, respectively. Among a group of vectors, a vector with a low $d$-component is preferred because a large $d$-component causes rapid flux variations and increases the torque ripple. Although the torque ripple can be decreased by increasing the switching frequency, it is not desirable due to the loss involved. Vectors with a low $d$-component are shown in Fig. 5 . The proposed switching sequence will be as Table I in which the small-, medium- and large vectors are selected to reduce, keep constant or increase the torque, respectively. The TI and FI indexes are related to the torque and flux errors. A value of ' 1 ' or ' -1 ', respectively, means that the desired quantity is exceeded and ' 0 ' means that it is within the range. Similarly,

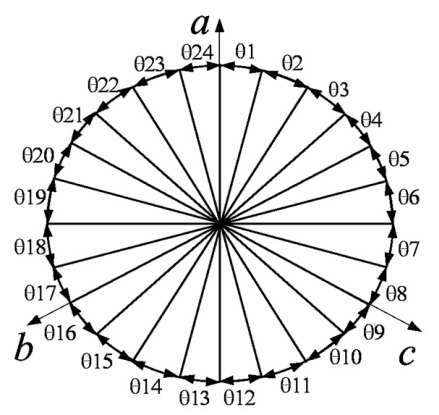

Fig. 4. 24 sectors in a multilevel inverter.

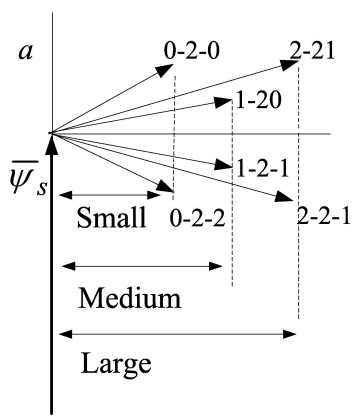

Fig. 5. Selected vectors based on low $d$-component in sector $\theta_{1}$.

the tables for other speeds can be provided.

\section{Overvoltage Problem at the Stator Terminal}

According to the reflection theory, the peak overvoltage created at the motor terminals is given by [15]:

$$
V_{r}=(1+\Gamma) V_{\text {pulse }}
$$

where $\Gamma$ denotes the reflection coefficient and can be calculated by:

$$
\Gamma=\frac{Z_{L}-Z_{o}}{Z_{L}+Z_{o}}
$$

where $Z_{L}$ and $Z_{o}$, respectively, denote the motor impedance and the cable characteristic impedance at high frequencies:

$$
Z_{o}=\sqrt{L_{o} / C_{o}}
$$

The parameters $L_{o}$ and $C_{o}$ are, respectively, the cable inductance and the cable capacitance per unit of length. If the motor is modeled by a simple RL model, it can be treated as an open circuit at high frequencies. Thus, the reflection coefficient will be nearly 1 . This implies a peak overvoltage $2 \mathrm{~V}$ pulse at the motor terminals. In [16] an analytical expression to calculate the reflection coefficient is extracted:

$$
\begin{aligned}
& \Gamma=\sin \left(\theta_{v}\right) / \theta_{v} \\
& \theta_{v}=v t_{r} / 2 l_{c}
\end{aligned}
$$

where, $l_{c}$ and $t_{r}$ are the cable length and the pulse rise time, respectively, and $v$ denotes the pulse propagation speed through the cable (32\% to $70 \%$ the speed of light in vacuum [11]). An idiomatic error in the literatures is that "multilevel drives reduce $d v / d t$ ". However, as can be seen in Fig. 6, multilevel drives do not reduce $d v / d t$. Indeed, multilevel inverters do not affect $\Gamma$ in equation (8) but they do reduce the size of $V_{\text {pulse. }}$ As mentioned earlier, one of the advantages of multilevel inverters is that the input voltage is divided by 
TABLE I

24-SECTOR SWITCHING TABLE AT HIGH SPEEDS

\begin{tabular}{|c|c|c|c|c|c|c|c|c|c|c|c|c|c|c|c|c|c|c|c|c|c|c|c|c|c|}
\hline & FI & 1 & 2 & 3 & 4 & 5 & 6 & 7 & 8 & 9 & 10 & 11 & 12 & 13 & 14 & 15 & 16 & 17 & 18 & 19 & 20 & 21 & 22 & 23 & 24 \\
\hline 1 & 1 & $0-2-2$ & $0-2-2$ & $0-1-2$ & $0-1-2$ & $00-2$ & $00-2$ & $-10-2$ & $-10-2$ & $-20-2$ & $-20-2$ & $-20-1$ & $-20-1$ & -200 & -200 & $-2-10$ & $-2-10$ & $-2-20$ & $-2-20$ & $-1-20$ & $-1-20$ & $0-20$ & $0-20$ & $0-2-1$ & $0-2$ \\
\hline 1 & -1 & $0-2-1$ & $0-2-1$ & $2-2$ & $0-2-2$ & $0-1-2$ & )-1-2 & 00-2 & $00-2$ & $-10-2$ & $-10-2$ & $-20-2$ & $-20-2$ & $-20-1$ & $-20-1$ & -200 & -200 & $-2-10$ & $-2-10$ & $2-20$ & $-2-20$ & $-1-20$ & $1-20$ & $0-20$ & \\
\hline 0 & 1 & $1-2-1$ & $1-2-2$ & $1-2$ & $1-1-2$ & $10-2$ & $11-2$ & $01-2$ & $01-2$ & $-11-2$ & $-21-2$ & $-21-1$ & 21-1 & -210 & -211 & -201 & -201 & $-2-11$ & $-2-21$ & $-1-21$ & $-1-21$ & -21 & $1-21$ & $1-20$ & \\
\hline 0 & -1 & $1-20$ & $1-2-1$ & $1-2-2$ & $1-2-2$ & $1-1-2$ & $10-2$ & $11-2$ & $11-2$ & $01-2$ & $-11-2$ & $-21-2$ & $-21-2$ & $-21-1$ & -210 & -211 & -211 & $\mid-201$ & $-2-11$ & $\mid-2-21$ & $-2-21$ & $-1-21$ & $0-21$ & $1-21$ & $1-2$ \\
\hline & 1 & $2-2-1$ & $2-2-2$ & $2-1-2$ & $20-2$ & $21-2$ & $22-2$ & $12-2$ & $02-2$ & $-12-2$ & $-22-2$ & $-22-1$ & -220 & -221 & -222 & -212 & -202 & $-2-12$ & $-2-22$ & $-1-22$ & $0-22$ & $1-22$ & $2-22$ & $2-21$ & $2-2$ \\
\hline & & $2-21$ & $2-20$ & $2-2-1$ & $2-2-2$ & $-1-2$ & $20-2$ & $1-2$ & $22-2$ & $12-2$ & $02-2$ & $-12-2$ & $22-2$ & $-22-1$ & -220 & -221 & -222 & -212 & -202 & $-2-12$ & $-2-22$ & $1-22$ & $0-22$ & $1-22$ & $2-2$ \\
\hline
\end{tabular}

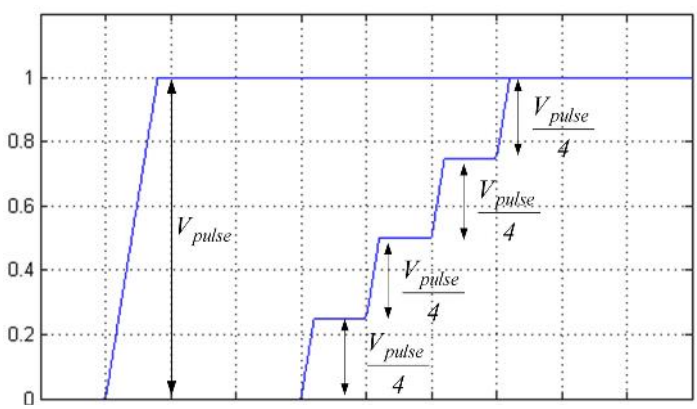

Fig. 6. Output voltage in a two- and a five- level inverter.

the number of levels and in every instant a fraction of the DC voltage is switched. Therefore, the peak overvoltage will be reduced as can be seen by comparing Fig. 7 and Fig. 8. However, the control strategy should make only one-level transitions at the output voltage otherwise this advantage will be lost. The applied $d v / d t$ is more important when the output voltage leads to $1 \mathrm{pu}$ because, for instance, output transition from $2 \mathrm{~V}_{\text {pulse }} / 4$ to $\mathrm{V}_{\text {pulse }}$ will cause the peak overvoltage to be $\left(1.5 \mathrm{~V}_{\text {pulse }}\right)$ and it is far more important than the transition from $\mathrm{V}_{\text {pulse }} / 4$ to $3 \mathrm{~V}_{\text {pulse }} / 4$, which causes $\left(1.25 \mathrm{~V}_{\text {pulse }}\right)$. The voltagelevel transition from the 0 level to $2 \mathrm{~V}_{\text {pulse }} / 4$ creates a peak overvoltage $\mathrm{V}_{\text {pulse }}$ that is not a detrimental overvoltage.

According to Table I column 3 (from the left related to sector1) when the vector changes from $0-2-2$ to $2-2-1$ (TI $=1$ to $\mathrm{TI}=-1$ ), a two-step transition will be applied to the stator. Therefore, the overvoltage will correspond to the reflection from a two-steps transition. These changes can be reduced using the redundancy of the vectors in which adding one voltage level to each phase does not affect the differential mode voltage.

For example, the vector $0-2-2$ has a redundant vector 11-1 which is the same in a differential mode. However, the output transition from 1-1-1 to 2-2-1 will be subjected to only a one-step transition in each phase. In the same way, the other two-level transitions could be replaced but not the vectors on the outer hexagon because they do not have redundancy. Finally, Table II is obtained. According to [17], over-voltages with a magnitude of 3-4pu bus voltage will occur where the last transient-overvoltage has not fully decayed before the application of the next pulse. In this paper, by limiting the switching frequency the occurrence of these over-voltages is prevented.

\section{CMV AND BEARING CURRENT}

Common-mode voltage (CMV) is defined as:

$$
V_{c o m}=\left(V_{a o}+V_{b o}+V_{c o}\right) / 3
$$
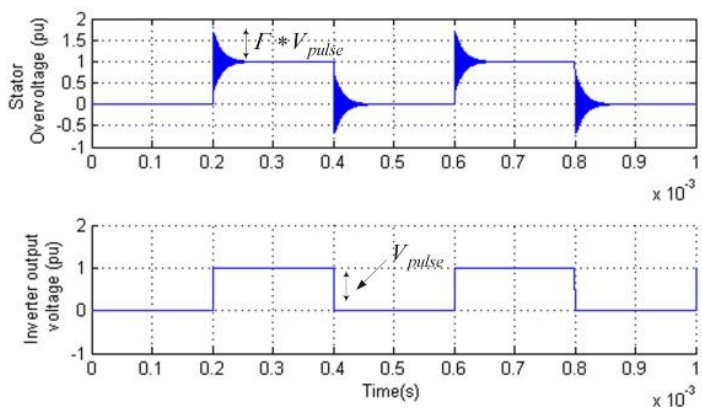

Fig. 7. Two-level Inverter and reflected voltage (pu)
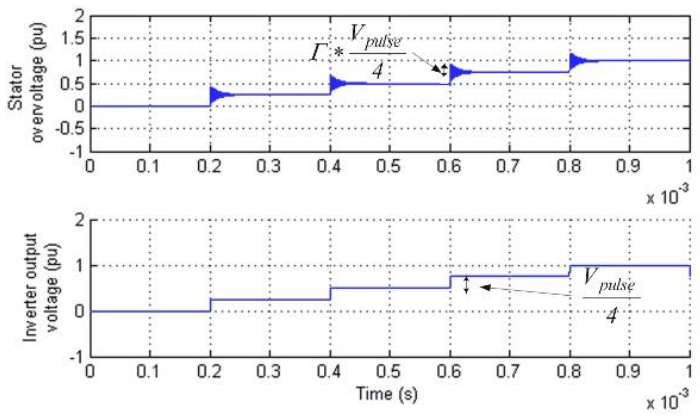

Fig. 8. Five-level Inverter and reflected voltage (pu).

Although there are several methods to reduce CMV, one interesting approach in multilevel inverters is to select voltage vectors that generate a zero- or low CMV at the load [17][20]. However, the main criterion in the bearing corrosion is the current density $j_{b}$ defined as:

$$
j_{b}=\frac{B V R \bullet V_{\text {com }}}{R_{b} \bullet A_{H}}
$$

where the BVR (Bearing Voltage Ratio) is a fraction of the CMV that drops across the bearing and is less than 0.1 [21]. Also, $\mathrm{Rb}$ is the oil electrical resistance after breakdown, about $10 \mathrm{ohms}$, and AH denotes the Hertzian contact area which is proportional to the square of the motor size $\left(\mathrm{AH} \approx \mathrm{h}^{2}\right)$. Although any voltage higher than the breakdown voltage leads to breakdown, the corrosion volume per discharge is determined as a function of the voltage squared [21]. Therefore, CMV reductions always reduce corrosion and increases bearing life. Also, a reduction of the CMV repetition rate (the number of discharges per sec.) has a similar effect.

According to Fig. 1, CMVs are measured with respect to the reference point ' $\mathrm{o}$ '. In practice this point is not grounded but it forms the common mode path at high frequencies through $Z_{\text {in }}$ (due to stray capacitance between the point ' $\mathrm{O}$ ' and the grounded frame). As mentioned earlier, using the voltage vector redundancy (adding one level or two levels to each phase) does not affect the differential mode voltage. 


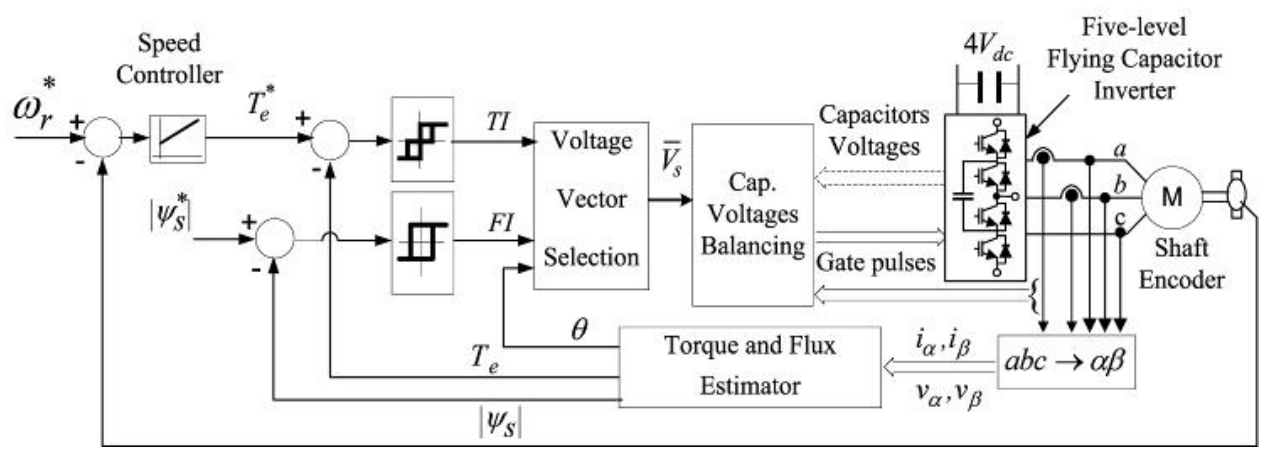

Fig. 9. DTC structure in a flying capacitor inverter.

TABLE II

SWITCHING TABLE AT HIGH-SPEEDS USING REDUNDANT VECTORS FOR TI=1

\begin{tabular}{|c|c|c|c|c|c|c|c|c|c|c|c|c|c|c|c|c|c|c|c|c|c|c|c|c|c|}
\hline & F & 1 & 2 & 3 & 4 & 5 & 6 & & & 9 & 10 & 11 & 12 & 13 & 14 & 15 & 16 & 17 & & 19 & 20 & 21 & 22 & 23 & \\
\hline & 1 & $-1-1$ & $-1-1$ & & 10-1 & $1-1$ & $11-1$ & $\overline{11-1}$ & & $11-1$ & & & & & -111 & -101 & -101 & $-1-11$ & & $0-11$ & & & & & \\
\hline & -1 & & & & & & & & & & & & & & & & & & & & & & & & \\
\hline & 1 & & & & & & & & & & & & & & & & & & & & & & & & \\
\hline & & & & & & & & & & & & & & & & & & & & & & & & & \\
\hline & 1 & & $2-2$ & $1-2$ & 0 & & $22-2$ & & $2-2$ & $2-2$ & $22-2$ & & -2 & & -2 & -212 & -202 & & $2-$ & $1-2$ & & & & & \\
\hline & & & & & & & & & & $2-2$ & $2-2$ & $2-2$ & 2 & & & & & & & & & & & & \\
\hline
\end{tabular}

TABLE III

CMV DUE TO DIFFERENT VECTORS $\left(1 \mathrm{PU}=4 \mathrm{~V}_{\mathrm{DC}}\right)$

\begin{tabular}{|c|c|c|c|}
\hline & Applied vector & $\begin{array}{c}\text { CMV } \\
\text { Before } \\
\text { Redundancy }\end{array}$ & $\begin{array}{c}\text { Reduced } \\
\text { CMV after } \\
\text { redundancy }\end{array}$ \\
\hline 1 & $2-22,-222,22-2$ & $1 / 6 \mathrm{pu}$ & $1 / 6 \mathrm{pu}$ \\
\hline 2 & $1-22,21-2,12-2,2-21,-212,-221$ & $1 / 12 \mathrm{pu}$ & $1 / 12 \mathrm{pu}$ \\
\hline 3 & $\begin{array}{c}0-22,2-20,20-2,02-2,-202 \\
1-21,-211,11-2\end{array}$ & 0 & 0 \\
\hline \multirow{2}{*}{4} & $\begin{array}{c}-1-22,2-1-2,2-2-1,-12-2,-22-1,-2-12 \\
-201,-210,10-2,1-20,0-21,01-2\end{array}$ & $-1 / 12 \mathrm{pu}$ & $-1 / 12 \mathrm{pu}$ \\
\hline \multirow{2}{*}{5} & $-2-22,2-2-2,-22-2$ & & $-1 / 6 \mathrm{pu}$ \\
\cline { 2 - 3 } & $\begin{array}{c}-1-21,-11-2,1-2-1,1-1-2,-2-21,-21-1 \\
0-20,00-2,-200\end{array}$ & $-1 / 6 \mathrm{pu}$ & $1 / 12 \mathrm{pu}$ \\
\cline { 2 - 3 } 6 & $\begin{array}{c}-2-21,-21-2,1-2-2,-1-1-1 \\
6\end{array}$ & $-1 / 4 \mathrm{pu}$ & $1 / 12 \mathrm{pu}$ \\
\hline 7 & $\begin{array}{c}-1-2-20,0-2-2,-20-2 \\
7\end{array}$ & $-1 / 3 \mathrm{pu}$ & 0 \\
\hline 8 & $-1-2-2,-1-2-1,-2-1-1-2,-2-2-1$ & $-5 / 12 \mathrm{pu}$ & $-1 / 12 \mathrm{pu}$ \\
\hline 9 & $-2-2-2$ & $1 / 2 \mathrm{pu}$ & $1 / 12 \mathrm{pu}$ \\
\hline
\end{tabular}

However, it changes the CMV and can be used to reduce the CMV. According to (12), by adding one or two levels to each phase, the CMV can be increased by $\mathrm{V}_{\mathrm{dc}}$ and $2 \mathrm{~V}_{\mathrm{dc}}$, respectively. Finally, the CMVs before- and after using the vector redundancy are shown in Table III. According to this table, in order to decrease the CMV, a two-level voltage is added to each phase in rows of 8 and 9 and a one-level voltage is added to each phases in rows 5, 6 and 7. Therefore, the largest CMV is related to the vectors -2-22, 2-2-2, -22$2,2-22,-222$ and $22-2$ which do not have redundant vectors so there is no possibility of reducing the CMV. The control strategy can ignore these vectors. Considering the high density of the vectors in a five-level drive, the removal of these vectors, which are located in the over-modulation area, will not reduce the drive capability significantly. Substituting the redundant vectors in Table II, Table IV is obtained in which the maximum CMV is $1 / 12 \mathrm{pu}\left(1 \mathrm{pu}=4 \mathrm{~V}_{\mathrm{dc}}\right)$.

\section{Simulation Results}

The DTC strategy shown in Fig. 9 is simulated and implemented using a prototype 400 -volt, $3 \mathrm{~kW}$ induction motor. The DTC and motor parameters are presented in the Appendix. To investigate the DTC performance with different tables, the following scenario is employed. The speed reference is set at $0.85 \mathrm{pu}$ and at $\mathrm{t}=0.15 \mathrm{~s}$, then a load torque of $0.5 \mathrm{pu}$ is applied. The stator current, rotor speed and electromagnetic torque using Table I and Table IV are shown in Fig. 10 and Fig. 11, respectively.

Before the DTC drive begins to generate electromagnetic torque, the stator flux is established by applying a constant voltage vector. During that the stator current increases above 2pu (3pu in peak). There is no significant difference between the waveforms in Fig. 10 and Fig. 11. This means that the drive performance is the same using both tables. The stator over-voltages using table I and table IV shown in Fig. 12 and Fig. 13, respectively, show different amplitudes. There is a $40 \%$ reduction due to the use of only a one-step addition to the phase voltage. The resulting CMVs are shown in Fig. 14 and Fig. 15. The CMV in Fig. 14 is between $-1 / 3$ pu and $1 / 6 \mathrm{pu}$. In other words, the maximum amplitude is $1 / 3 \mathrm{pu}$. The CMV using table IV shown in Fig. 15 is between $1 / 12 \mathrm{pu}$ and $-1 / 12 \mathrm{pu}$, which means a $75 \%$ reduction. In addition, the repetition frequency is reduced and therefore the bearing life will be increase significantly. With a $600 \mathrm{~V}$ DC bus, the $\mathrm{CMV}$ value is $600 \mathrm{~V} / 12=50 \mathrm{~V}$. On the other hand, the bearing voltage ratio (BVR) is smaller than 0.1 and therefore the rotor shaft voltage is less than the breakdown voltage at the bearings. In medium voltage motors, although the CMV is higher due to increasing the Hertzian contact area, the bearing current density is small and will rarely cause corrosion of the bearings. 
TABLE IV

SWITCHING TABLE FOR HIGH SPEEDS USING REDUNDANT VECTORS FOR TI=0

\begin{tabular}{|c|c|c|c|c|c|c|c|c|c|c|c|c|c|c|c|c|c|c|c|c|c|c|c|c|c|}
\hline TI & FI & 1 & 2 & 3 & 4 & 5 & 6 & 7 & 8 & 9 & 10 & 11 & 12 & 13 & 14 & 15 & 16 & 17 & 18 & 19 & 20 & 21 & 22 & 23 & 24 \\
\hline 1 & 1 & $1-1-1$ & $1-1-1$ & $10-1$ & $10-1$ & $11-1$ & $11-1$ & $1-1$ & $01-1$ & $-11-1$ & $-11-1$ & -110 & -110 & -111 & -111 & -101 & -101 & $-1-11$ & $-1-11$ & $0-11$ & $0-11$ & $1-11$ & $1-11$ & $1-10$ & $1-10$ \\
\hline 1 & -1 & $1-10$ & $1-10$ & $1-1-1$ & $1-1-1$ & $10-1$ & $10-1$ & $11-1$ & $11-1$ & $01-1$ & $01-1$ & $-11-1$ & $-11-1$ & -110 & -110 & -111 & -111 & -101 & -101 & $-1-11$ & $-1-11$ & $0-11$ & $0-11$ & $1-11$ & $1-11$ \\
\hline 0 & 1 & $2-10$ & $2-1-1$ & $2-1-1$ & $2-1-1$ & $10-2$ & $11-2$ & $01-2$ & $01-2$ & $02-1$ & $-12-1$ & -120 & -120 & -210 & -211 & -201 & -201 & -102 & $-1-12$ & $0-12$ & $0-12$ & $0-21$ & $1-21$ & $1-20$ & $1-20$ \\
\hline 0 & -1 & $1-20$ & $2-10$ & $2-1-1$ & $2-1-1$ & $20-1$ & $10-2$ & $11-2$ & $11-2$ & $01-2$ & $02-1$ & $-12-1$ & $-12-1$ & -120 & -210 & -211 & -211 & -201 & -102 & $-1-12$ & $-1-12$ & $0-12$ & $0-21$ & $1-21$ & $1-21$ \\
\hline-1 & 1 & $2-2-1$ & $2-1-2$ & $2-1-2$ & $20-2$ & $21-2$ & $12-2$ & $12-2$ & $02-2$ & $-12-2$ & $-22-1$ & $-22-1$ & -220 & -221 & -212 & -212 & -202 & $-2-12$ & $-1-22$ & $-1-22$ & $0-22$ & $1-22$ & $2-21$ & $2-21$ & $2-20$ \\
\hline-1 & -1 & $2-21$ & $2-20$ & $2-2-1$ & $2-2-1$ & $2-1-2$ & $20-2$ & $21-2$ & $21-2$ & $12-2$ & $02-2$ & $-12-2$ & $-12-2$ & $-22-1$ & -220 & -221 & -221 & -212 & -202 & $-2-12$ & $-2-12$ & $-1-22$ & $0-22$ & $1-22$ & $1-22$ \\
\hline
\end{tabular}
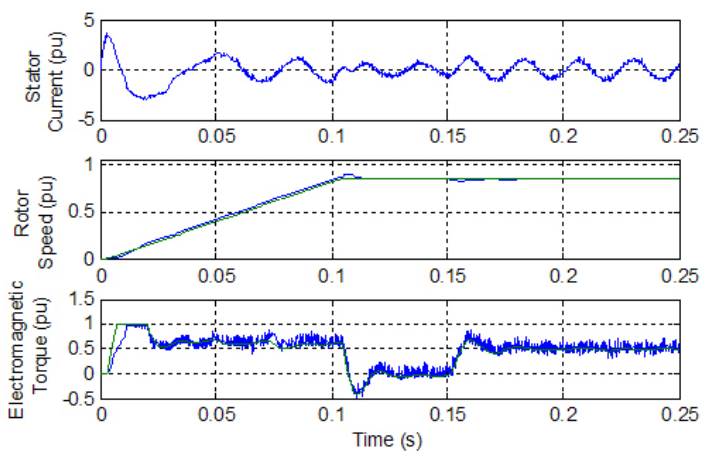

Fig. 10. Waveforms using Table I.
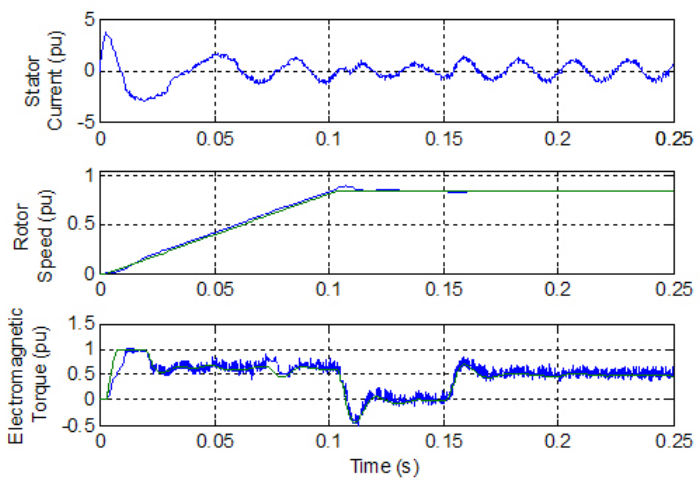

Fig. 11. Waveforms using Table IV.

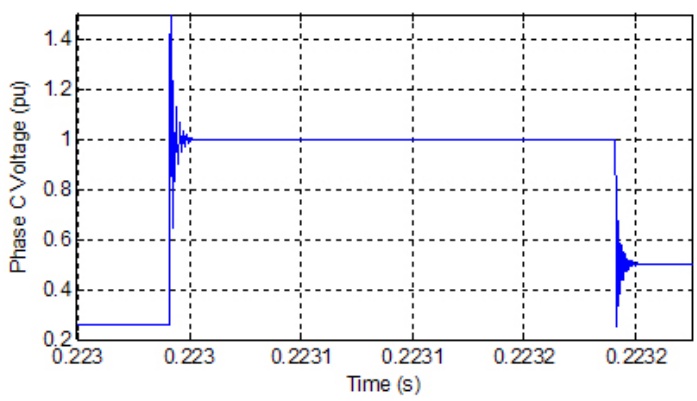

Fig. 12. Overvoltage using Table I.

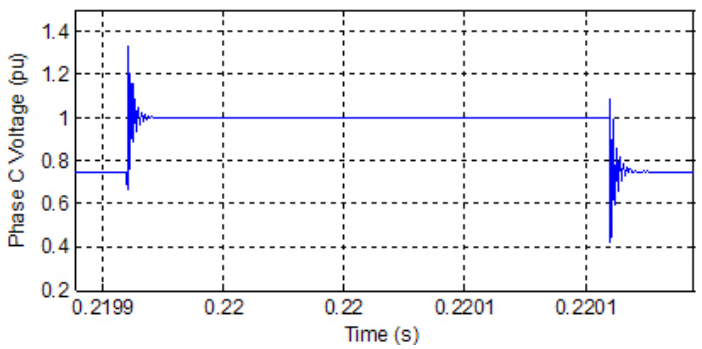

Fig. 13. Overvoltage using Table IV.

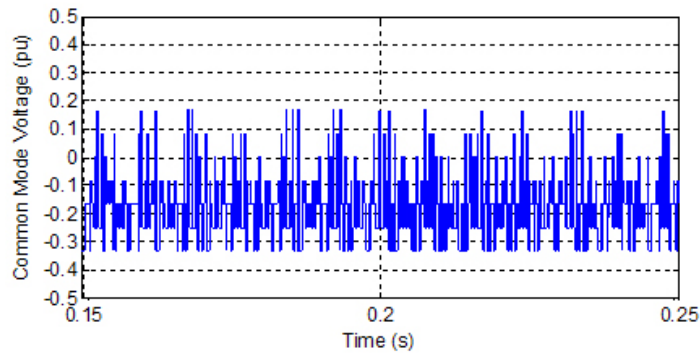

Fig. 14. CMV using Table I.

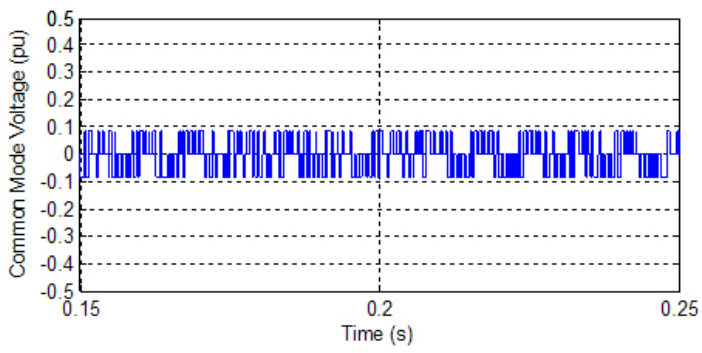

Fig. 15. CMV using Table IV.

\section{PRACTICAL RESUltS}

A prototype five-level flying capacitor drive was built and is shown in Fig. 16. Different parts of the system have been named including the IGBTs and associated drivers, the flying capacitors, the voltage transducers and the DSP controller. The main processor board, an ezDSPF2812 with 150MIPS capability, is suitable for implementing the DTC. The processor is equipped with 16 channel ADC-converters which are used to measure nine floating-capacitor voltages, two phase-currents, three phase-voltages and the DC link voltage. Six I/O ports with 56 pins provide the gate commands to the 24 IGBTs easily. To measure the capacitor voltages, isolated DC-voltage transducers are used. The DC generator shown in Fig. 17 is used as a mechanical load. The amount of load is adjustable by heating elements. The induction motor is accelerated to $85 \%$ of the nominal speed using Tables I and IV, and the results are shown in Fig. 18 and 19. In the several experiments that were repeated, there is always less noise in the measured speed using table IV. The authors believe that this is due to a reduction of the EMI caused by the CMV reduction. The peak overvoltage in Fig. 21 as compared with Fig. 20 confirms about a 50\% reduction. The CMV reduction in Fig. 23 is evident when compared to Fig. 22.

\section{CONCLUSION}

In this paper, a 24-sector DTC strategy for a five-level flying capacitor drive is analyzed and a proper switching table 


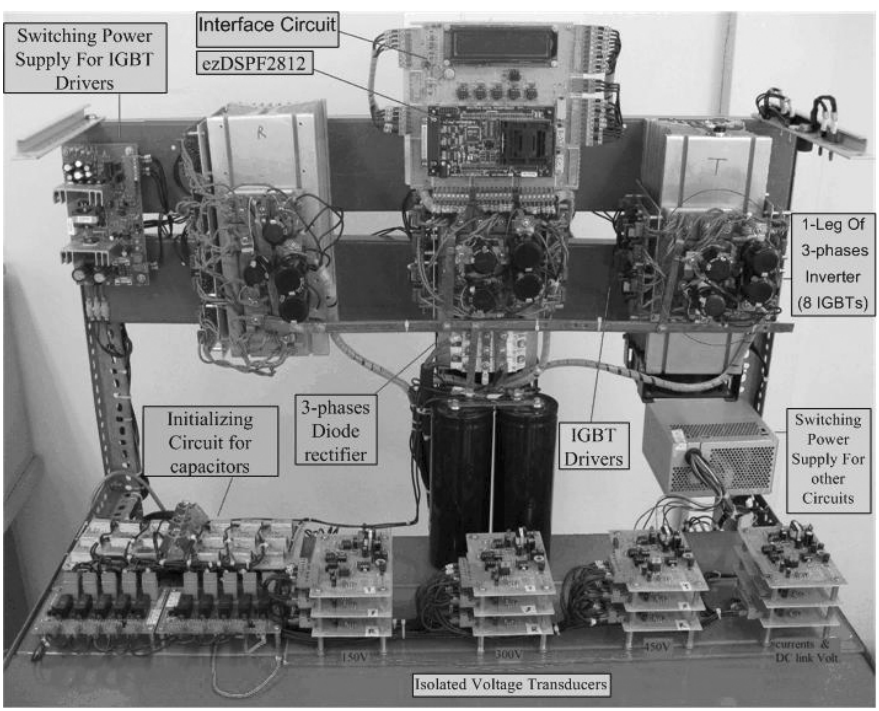

Fig. 16. Implemented system.

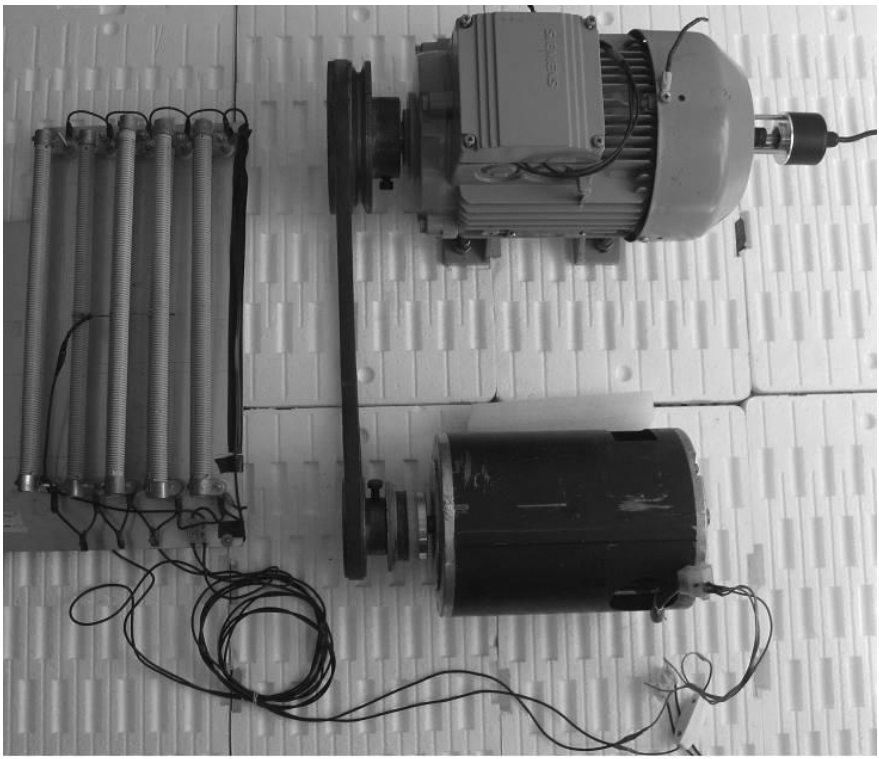

Fig. 17. DC generator as mechanical load.

is presented. Then the switching table is modified to reduce the output $\mathrm{dv} / \mathrm{dt}$ by $40 \%$ and the CMV to $25 \%$ of its initial value. Simulation and practical results obtained from the DTC using a prototype $400 \mathrm{~V}, 3 \mathrm{kVA}$ system confirm the proposed improvements.

\section{APPENDIX}

\section{Induction motor specifications:}

$\mathrm{V}=400 \mathrm{~V}$, Power $=3 \mathrm{~kW}, \mathrm{p}=2$ (4 pole), $\mathrm{Rs}=1.873 \Omega, \mathrm{Rr}=1.86 \Omega$,

Lls=Llr $=7.54 \mathrm{mH}, \mathrm{Lm}=210 \mathrm{mH}, \mathrm{J}=0.01 \mathrm{~kg}-\mathrm{m} 2$.

Direct torque control parameters:

Torque hysteresis Band-width $=0.2 \mathrm{~N} . \mathrm{m}$,

Flux hysteresis Band-Width $=0.01 \mathrm{~Wb}$,

Initial and nominal machine flux $=0.8 \mathrm{~Wb}$

Maximum switching frequency $=5000 \mathrm{~Hz}$,

DTFC sampling $=40 \mu \mathrm{S}$

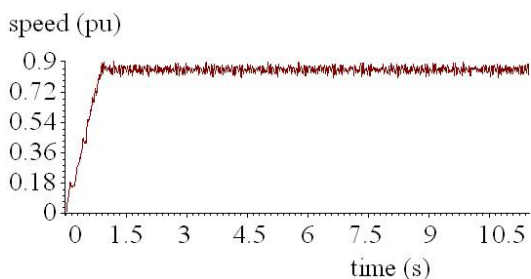

Fig. 18. Speed variation using Table I.

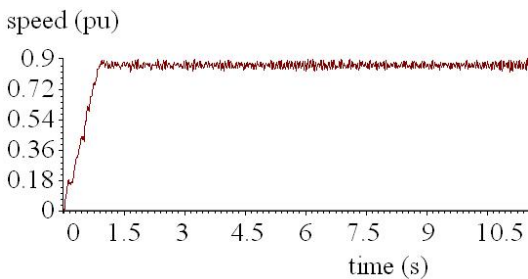

Fig. 19. Speed variation using Table IV.

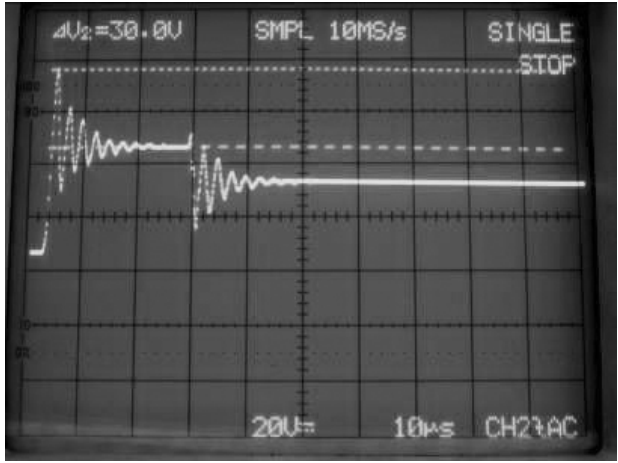

Fig. 20. Overvoltage using Table I, volts $/ \mathrm{div}=200 \mathrm{~V}$, time $/ \mathrm{div}=10 \mu \mathrm{s}$.

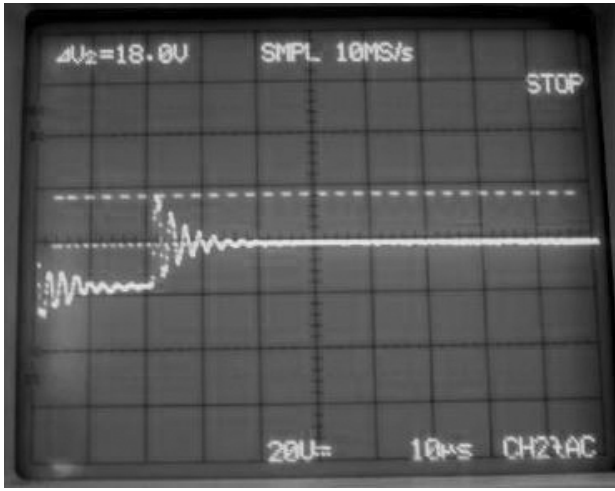

Fig. 21. Overvoltage using Table IV, volts $/ \mathrm{div}=200 \mathrm{~V}$, time $/ \mathrm{div}=10 \mu \mathrm{s}$.

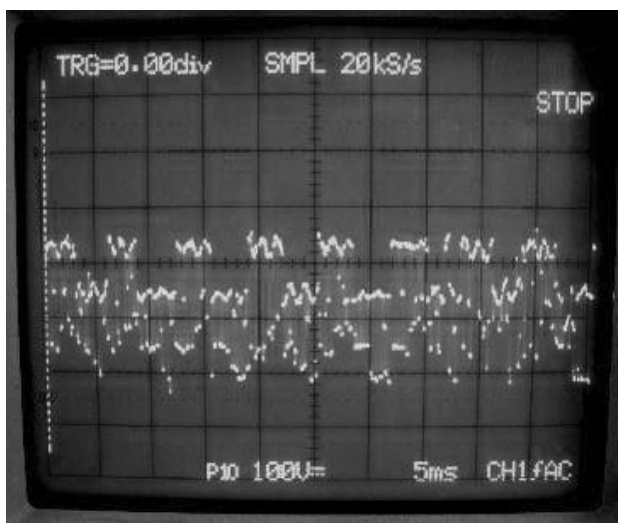

Fig. 22. CMV using Table I, volts $/ \mathrm{div}=100 \mathrm{~V}$, time $/ \mathrm{div}=5 \mathrm{~ms}$. 


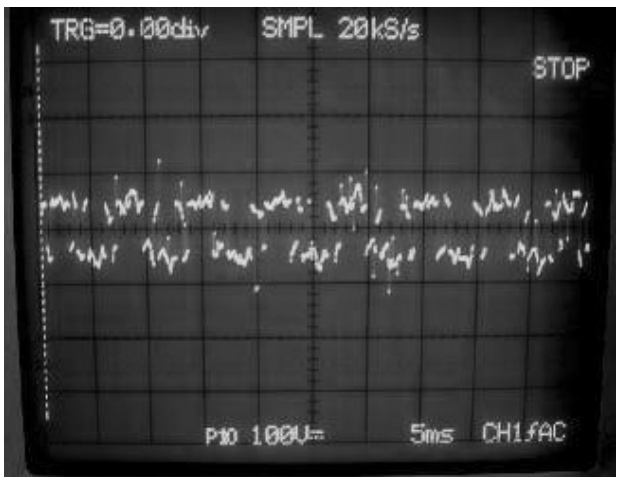

Fig. 23. CMV using Table IV, volts/div=100V, time/div=5ms.

\section{REFERENCES}

[1] J. Rodr?guez, S. Bernet, BinWu, J. O. Pontt, and S. Kouro, "Multilevel voltage-source-converter topologies for industrial medium-voltage drives," IEEE Trans. Indust. Elec., Vol. 54, No. 6, pp. 2930-2945, Dec. 2007.

[2] S. Kouro, M. Malinowski, K. Gopakumar, J. Pou, L. G. Franquelo, B. Wu, J. Rodriguez, M. A. P?rez, and J. I. Leon, "Recent advances and industrial applications of multilevel converters," IEEE Trans. Indust. Elec., Vol. 57, No. 8, pp. 2553-2580, Aug. 2010.

[3] H. Abu-Rub, J. Holtz, J. Rodriguez, and G. Baoming, "Medium-voltage multilevel converters-State of the art, challenges, and requirements in industrial applications," IEEE Trans. Indust. Elec., Vol. 57, No. 8, pp. 2581-2596, Aug. 2010.

[4] S. S. Fazel, S. Bernet, D. Krug, and K. Jalili, "Design and comparison of 4-kV neutral-point-clamped, flying-capacitor, and series-connected $\mathrm{H}$ bridge multilevel converters," IEEE Trans. Ind. Appl., Vol. 43, pp. 10321040, Jul./Aug. 2007.

[5] D. Krug, S. Bernet, S. S. Fazel, K. Jalili, and M. Malinowski, "Comparison of $2.3-\mathrm{kV}$ medium-voltage multilevel converters for industrial medium-voltage drives," IEEE Trans. Indust. Elec., Vol. 54, No. 6, pp. 2979-2992, Dec. 2007.

[6] J. Rodr?guez, J. Pontt, S. Kouro, and P. Correa, "Direct torque control with imposed switching frequency in an 11-level cascaded inverter," IEEE Trans. on Indust. Elec., Vol. 51, pp. 827-833, Aug. 2004.

[7] A. Sapin, P. K. Steimer, and J.-J. Simond, "Modeling, simulation, and test of a three-level voltage-source inverter with output LC filter and direct torque control," IEEE Trans. Ind. Appl., Vol. 43, No. 2, pp. 492498, Mar./Apr. 2007.

[8] C. A. Martins, X. Roboam, T. A. Meynard, A. S. Carvalho, "Switching frequency imposition and ripple reduction in DTC drives by using a multilevel converter," IEEE Trans. Power Electron., Vol. 17, No. 2, pp. 286-297, Mar. 2002.

[9] M. F. Escalante, J.-C. Vannier and A. Arzande, "Flying capacitor multilevel inverters and DTC motor drive applications," IEEE Trans. Indust. Elec., Vol. 49, pp.809-815, Aug. 2002.

[10] E. Persson, "Transient effects in application of PWM inverters to induction motors," IEEE Trans. Ind. Applicat., Vol. 28, pp. 1095-1101, Sep./Oct. 1992.

[11] R. Kerkman, D. Leggate, and G. Skibinski, "Interaction of drive modulation and cable parameters on AC motor transients," IEEE Trans. Ind. Appl., Vol. 33, No. 3, pp. 722-731, May/Jun. 1997.

[12] J. Rodr?guez, J. Pontt, C. Silva, R. Musalem, P. Newman, R. Vargas, and S. Fuentes, "Resonances and overvoltages in a medium-voltage fan motor drive with long cables in an underground mine," IEEE Trans. Ind. Appl., Vol. 42, No. 3, pp. 856-863, May/Jun. 2006.

[13] F. Endrejat, and P. Pillay, "Resonance overvoltages in medium-voltage multilevel drive systems," IEEE Trans. Ind. Appl., Vol. 45, No. 4, pp. 1199-1209, Jul./Aug. 2009.

[14] J. Erdman, R. Kerkman, and D. Schlegel, "Effect of PWM inverters on AC motor bearing currents and shaft voltages," IEEE Trans. Ind. Appl., Vol. 32, No. 2, pp. 250-259, Mar./Apr. 1996.
[15] E. J. Bartolucci, and B. H. Finke, "Cable design for PWM variablespeed AC drives," IEEE Trans. Ind. Appl., Vol. 37, No. 2, pp. 245-252, Mar./Apr. 2001

[16] T. R. Mukundan, "Calculation of voltage surges on motors fed from pwm drives-A simplified approach," IEEE Trans Energy Conver., Vol. 19, No. 1, pp. 223-225, Mar. 2004.

[17] G. Skibinski, D. Leggate, and R. Kerkman, "Cable characteristics and their influence on motor over-voltages," in Proc. IEEE APEC, Vol. 1, pp. 114-121, 1997.

[18] H. Zhang, A. V. Jouanne, S. Dai, A. K. Wallace, and F. Wang, "Multilevel inverter modulation schemes to eliminate common-mode voltages," IEEE Trans. Ind. Appl., Vol. 36, pp. 1645-1653, Nov./Dec. 2000.

[19] W. Leonhard, Control of Electrical Drives, Third edition, Berlin, Springer, 2001.

[20] J. Rodr?guez, J. Pontt, P. Correa, P. Cort?s, and C. Silva, "A new modulation method to reduce common-mode voltages in multilevel inverters," IEEE Trans. Indust. Electron., Vol. 51, No. 4, Aug. 2004.

[21] A. Muetze and A. Binder, "Calculation of motor capacitances for prediction of the voltage across the bearings in machines of inverterbased drive systems," IEEE Trans. Ind. Appl., Vol. 43, No. 3, pp. 665672, May/Jun. 2007.

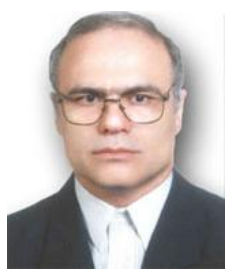

Abdolreza Rahmati was born in Abadeh, Iran, in 1951. He received his B.S. in Electronics Engineering from the Iran University of Science and Technology (IUST), Tehran, in 1979, and his M.S. and Ph.D. in Power Electronics from Bradford University, West Yorkshire, U.K., in 1987 and 1990, respectively. He is currently working as an Associate Professor at IUST Iran. His current research interests include microprocessor and microcontroller-based system design, motor drives and control, HVDC transmissions, modulation strategies for power electronic systems, multilevel inverters, and power devices. He is a Member of the Institution of Engineering and Technology (IET), and the Engineering Council, U.K.

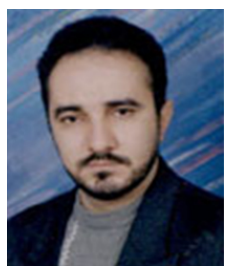

Mohammad Arasteh was born in Tehran, Iran, in 1968 $\mathrm{He}$ received his B.S. in Electronics Engineering from the Iran University of Science and Technology (IUST), in 1992, and his M.S. in Electronics Engineering from the University of Tehran, in 1995. He is currently working toward his Ph.D. at IUST, Iran. His current research interests include power electronics, multilevel drives and power quality.

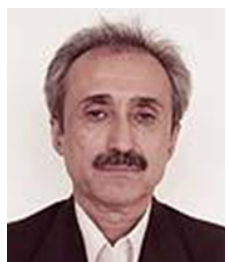

Shahrokh Farhangi obtained his B.S., M.S. and Ph.D. in Electrical Engineering from the University of Tehran, Iran, with honors. He is a Professor in the School of Electrical and Computer Engineering, University of Tehran. His research interests include the design and modeling of power electronic converters, drives, photovoltaics and renewable energy systems. He has published more than 100 papers in conference proceedings and journals. He has managed several research and industrial projects, some of which have won national and international awards. He was selected as a Distinguished Engineer in Electrical Engineering by the Iran Academy of Sciences, in 2008.

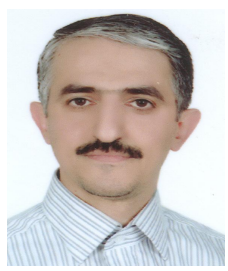

Adib Abrishamifar was born in Tehran, Iran, in 1967. $\mathrm{He}$ received his B.S., M.S. and Ph.D. in Electronics Engineering from the Iran University of Science and Technology (IUST), Iran, in 1989, 1992 and 2001, respectively. He has been with the Department of Electrical Engineering, IUST, since 1993. His current research interests include analog integrated circuit design and power electronics. 